\title{
OPEN Protein network analyses of pulmonary endothelial cells in chronic thromboembolic pulmonary hypertension
}

Sarath Babu Nukala ${ }^{1,8,9 凶}$, Olga Tura-Ceide ${ }^{3,4,5,9}$, Giancarlo Aldini ${ }^{1}$, Valérie F. E. D. Smolders ${ }^{2,3}$, Isabel Blanco ${ }^{3,4}$, Victor I. Peinado ${ }^{3,4}$, Manuel Castellà ${ }^{6}$, Joan Albert Barberà ${ }^{3,4}$, Alessandra Altomare ${ }^{1}$, Giovanna Baron ${ }^{1}$, Marina Carini ${ }^{1}$, Marta Cascante ${ }^{2,7,9}$ \& Alfonsina D'Amato ${ }^{1,9}$

Chronic thromboembolic pulmonary hypertension (CTEPH) is a vascular disease characterized by the presence of organized thromboembolic material in pulmonary arteries leading to increased vascular resistance, heart failure and death. Dysfunction of endothelial cells is involved in CTEPH. The present study describes for the first time the molecular processes underlying endothelial dysfunction in the development of the CTEPH. The advanced analytical approach and the protein network analyses of patient derived CTEPH endothelial cells allowed the quantitation of 3258 proteins. The 673 differentially regulated proteins were associated with functional and disease protein network modules. The protein network analyses resulted in the characterization of dysregulated pathways associated with endothelial dysfunction, such as mitochondrial dysfunction, oxidative phosphorylation, sirtuin signaling, inflammatory response, oxidative stress and fatty acid metabolism related pathways. In addition, the quantification of advanced oxidation protein products, total protein carbonyl content, and intracellular reactive oxygen species resulted increased attesting the dysregulation of oxidative stress response. In conclusion this is the first quantitative study to highlight the involvement of endothelial dysfunction in CTEPH using patient samples and by network medicine approach.

Chronic thromboembolic pulmonary hypertension (CTEPH) is a major complication of pulmonary embolism, characterized by an abnormal increase of pulmonary artery pressure due to the presence of organised thromboembolic material in pulmonary arteries that has not resolved after a period of appropriate anticoagulant therapy ${ }^{1}$. The progressive increase of pulmonary vascular resistance in CTEPH may ultimately lead to right ventricular failure and death ${ }^{1}$. CTEPH is a dual vascular disorder, that combines occluded pulmonary vessels and distal arteriopathy in non-occluded vessels. Inflammation, autoimmunity, and angiogenesis may also occur ${ }^{2,3}$. The mechanisms underlying the persistence of thrombotic lesions and distal arteriopathy are poorly understood. The vascular endothelium, constituted by a thin layer of endothelial cells (ECs), is at the interface between circulating blood and the vessel wall. It regulates homeostasis, platelet activity, leukocyte adhesion and thrombosis ${ }^{4,5}$. The

\footnotetext{
${ }^{1}$ Department of Pharmaceutical Sciences, Università Degli Studi Di Milano, 20133 Milan, Italy. ${ }^{2}$ Department of Biochemistry and Molecular Biomedicine and Institute of Biomedicine (IBUB), Faculty of Biology, University of Barcelona, Barcelona, Spain. ${ }^{3}$ Department of Pulmonary Medicine, Hospital Clínic-Institut D'Investigacions Biomèdiques August Pi I Sunyer (IDIBAPS), University of Barcelona, Barcelona, Spain. ${ }^{4}$ Centro de Investigación Biomédica en Red de Enfermedades Respiratorias (CIBERES), Instituto de Salud Carlos III (ISCIII), CB06/06/0011 Madrid, Spain. ${ }^{5}$ Department of Pulmonary Medicine, Dr. Josep Trueta University Hospital de Girona, Santa Caterina Hospital de Salt and the Girona Biomedical Research Institut (IDIBGI), Girona, Spain. ${ }^{6}$ Department of Cardiovascular Surgery, Institut Clínic del Tòrax, Hospital Clínic, University of Barcelona, Barcelona, Spain. ${ }^{7}$ Centro de Investigación Biomédica en Red de Enfermedades Hepáticas y Digestivas (CIBEREHD)CB17/04/00023 and Metabolomics Node At INB-Bioinfarmatics Platform, Instituto de Salud Carlos III (ISCIII), Madrid, Spain. ${ }^{8}$ Department of Pharmacology, The University of Illinois College of Medicine, 909 S Wolcott Ave, COMRB 4097, Chicago, IL 60612, USA. ${ }^{9}$ These authors contributed equally: SarathBabu Nukala, Olga Tura-Ceide, Marta Cascante and Alfonsina D'Amato ${ }^{\varpi}$ email: sarath.nukala@unimi.it; alfonsina.damato@unimi.it
} 
endothelium comprises adherents and tight junctions and expresses proteins, such as VE-cadherin in association with a-, b-, and g-catenins; platelet endothelial cell adhesion molecule 1 (PECAM-1); cortical actin filaments linked with zonula occludens-1 (ZO-1), vinculin and $\alpha$-actinin, involved in actin filament network. Oxidative stress causes phosphorylation and reorganization of occludin and PECAM-1, reduces levels of vascular endothelium catenins and actin-binding proteins, resulting in disruption of the tight and adherent junctions ${ }^{6}$.

Endothelial dysfunction plays a fundamental role in promoting thrombus formation and pulmonary vascular remodeling. In pathological conditions, the loss of endothelium homeostatic function induces the disorganization of the vascular wall and the consequent development of vascular lesions. E-selectin, intercellular adhesion molecule (ICAM)-1, tissue factor, and vascular endothelial growth factor (VEGF) receptors ${ }^{7}$ are specific markers of endothelium dysfunction. In addition $\mathrm{Kv}$ channels and the $\mathrm{Ca}^{2+}$ signal are dysregulated in the development of pulmonary vascular remodelling in patients with idiopathic pulmonary arterial hypertension (IPAH) ${ }^{8}$. Pulmonary endarterectomy (PEA) is the treatment of choice in CTEPH. The intravascular thrombotic material along with the adhered fibrotic endothelial layer is surgically removed. As a result, pulmonary hemodynamics improve dramatically, with a complete resolution of pulmonary hypertension in most cases $^{9-11}$. The location occlusive lesions in distal vessels in several patients precludes their surgical removal. In these cases, pharmacological treatment and/or balloon pulmonary angioplasty (BPA) are potential therapeutic alternatives ${ }^{12}$.

The physiological mechanisms underlying CTEPH are still unknown and the present study of in depth description of patient endothelium's phenotypes may help to get an early diagnosis and better prognosis of CTEPH disease. Label free quantitative proteomics approach and network analysis of differentially regulated proteins in patient-derived cells provide a unique opportunity to investigate the molecular mechanisms of the disease.

\section{Results}

Differentially regulated protein pathways in CTEPH. To characterize the potential molecular mechanisms underlying endothelial dysfunction in the development of the CTEPH, patient-derived CTEPH-ECs and healthy Human Pulmonary Artery Endothelial Cells (HPAECs) were analyzed by label free quantitative proteomics approach. 3258 proteins and 27,678 unique peptides were identified and quantified. 673 proteins were differentially regulated of which 82 proteins were overexpressed, and 232 proteins resulted down-regulated in the CTEPH-ECs compared to the HPAEs group, with a ratio's threshold of 1.5 (Fig. 1, Table 1, Suppl. Table 1). Procollagen-lysine, 2-oxoglutarate 5-dioxygenase $2($ PLOD2, ratio $=5.50)$ and Transgelin $($ TGLN, ratio $=5.28)$, proteins involved in the cross-linking respectively of collagen and actin, resulted highly overexpressed, indicating the presence of fibrosis in CTEPH ECs ${ }^{13,14}$.

Endoglin $(E N G$, ratio = 3.05), a vascular endothelium glycoprotein was upregulated. It is involved in the positive regulation of angiogenesis, response to hypoxia, positive regulation of collagen biosynthesis and negative regulation of nitric-oxide (NO) synthase processes. Nitric oxide synthase 3 was also highly down expressed $(\mathrm{NOS} 3$, ratio $=0.52)$ indicating low concentration of $\mathrm{NO}$ and consequent impairment of vascular remodelling ${ }^{15,16}$ (Table 1).

Von Willebrand Factor (vWF), an important adhesion protein involved in vascular haemostasis, was down regulated (ratio $=0.42$ ), as also validated by RT-PCR analyses (Suppl. Figure 1 ).

Cell movement and angiogenesis functional modules were predicted to be decreased ( $p$ value $7.40 \mathrm{e}^{-05}, 44$ genes, $\mathrm{z}$ score -2.994), thanks to the contribution of differentially regulated proteins, such as RALA (ratio $=0.52)$, ERAP2 (ratio=0.17) TPT1 (ratio=5.20), MCM2 (ratio=2.11) and CNN2 (ratio=2.17) (Fig. 2 and Suppl. Figure 2). The decrease of the calcium signalling pathway (z score -2.23 , p-value $2.73 \mathrm{e}^{-3}, 9$ genes) correlates with the decrease of PI3K/AKT, mTOR, NFAT, eiF4 and p70S6K pathways (Fig. 3, Suppl. Table 2). The differentially expressed proteins describing the pathways are directly involved in cell proliferation, essential in the remodelling of vascular endothelium. The regulation of these proteins, such as integrin or phosphatase activator isoforms, disagreed with expression in healthy samples in literature, attesting an overall dysregulation of these pathways in CTEPH disease.

Protein network analyses revealed a pronounced mitochondrial dysfunction (p-value $3.10 \mathrm{e}^{-29}, 34$ genes). Figure 4 shows the downregulated respiratory and oxidative phosphorylation pathways in mitochondria. Proteins belonging to complexes I, II and III, such as cytochrome $\mathrm{cl}(\mathrm{Cycl}$, ratio $=0.64)$, glutathione peroxidase $4(\mathrm{GPX} 4$, ratio $=0.22)$, NADH ubiquinone oxidoreductase core subunit A8 (NDUFA8, ratio $=0.43$ ) succinate dehydrogenase complex flavoprotein A (SDHA, ratio $=0.60)$ were down expressed. GPX4 is an essential antioxidant peroxidase that protect cells from oxidative damage by preventing membrane lipid peroxidation. However, in the present study GPX4 showed a drastic downregulation (ratio=0.2), suggesting the dysregulation of oxidative stress/damage response. Interestingly the metabolism of reactive oxygen species resulted decreased (z score -0.684, p-value $7.8 \mathrm{e}^{-10}, 8$ genes) (Fig. 5), showing an impaired response to oxidative stress, confirmed by down regulation of NRF2 pathway ( $\mathrm{z}$ score -1.342 , p-value $5.93 \mathrm{e}^{-3}, 34$ genes), principal actor in preventing oxo-damage (Fig. 3, Suppl. Table 2).

mRNA translation was up regulated, as resulted from the increase of EIF2 signaling pathway ( $\mathrm{p}$ value 1.00 $\mathrm{e}^{-11}, 21$ genes, $\mathrm{z}$ score 1.941) in which eukaryotic translation initiation factors and ribosomal proteins were over expressed. The down regulation of proteins, such as Lysosomal Pro-X carboxypeptidase (PRCP, ratio $=0.26)$ induced a decrease of angiogenesis functional module ( $\mathrm{z}$ score -0.389 , p-value $1.72 \mathrm{e}^{-05}$ ) and cell movement of leukocytes signaling ( $\mathrm{z}$ score -1.50 , p-value $\left.1.12 \mathrm{e}^{-04}\right)$, key components of thrombus resolution.

Fatty acid metabolism ( $\mathrm{z}$ score -0.933 , p-value $2.58 \mathrm{e}^{-10}, 38$ genes) was decreased while the concentration of lipids ( $\mathrm{z}$ score 1.846 , p-value $1.74 \mathrm{e}^{-6}, 39$ genes) was increased, attesting dysfunction in lipid metabolism during CTEPH disease (Suppl. Figure 3 and 4). In addition, inflammation of organ was predicted to be increased ( $z$ score 3.147, p-value $5.03 \mathrm{e}^{-05}, 24$ genes) (Suppl. Figure 5 and Suppl. Table 3). 


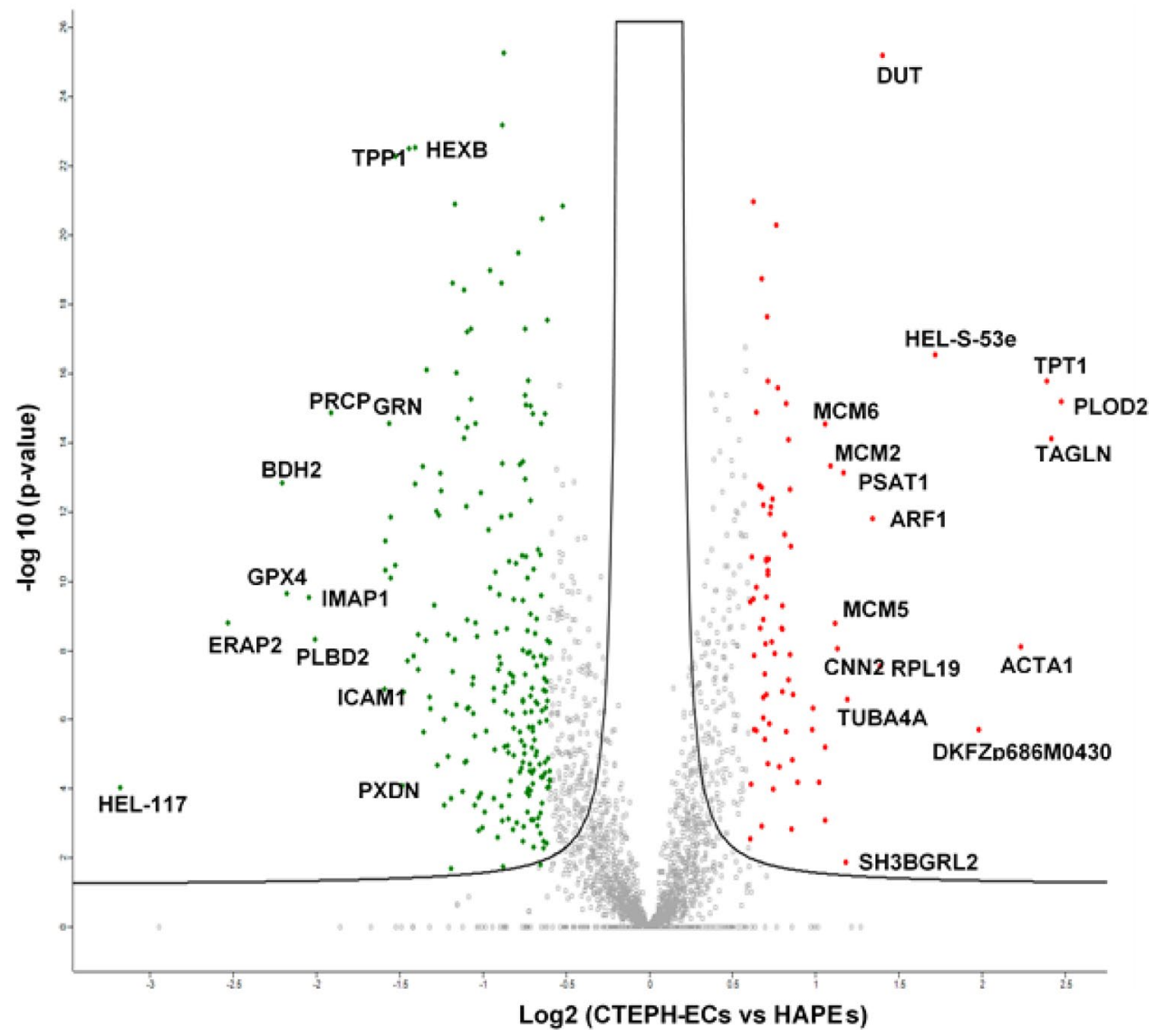

Figure 1. Distribution of altered proteins in CTEPH-ECs cells. In the above scatter plot, X-axis represents log2 ratio and $y$-axis represent $-\log \mathrm{p}$ value of significantly differentially regulated proteins. Green colour symbolizes down-regulation, red colour symbolizes upregulation.

To understand the status of oxidants, the levels of advanced oxidation protein products, (AOPPs), total protein carbonyl content (PCO), and intracellular reactive oxygen species (ROS) were quantified. Similarly, to study the status of antioxidant levels the GSH (reduced)/GSSG (oxidized) ratio, and NADPH (reduced)/NADP (oxidized) ratio was measured. As shown in Fig. 6, the levels of AOPPs, total PCOs, and intracellular ROS were increased in dysfunctional ECs of CTEPH, whereas GSH to GSSG and NADPH to NADP ratios were decreased.

\section{Discussion}

CTEPH is a multi-factorial disease and its diagnosis and treatment are complex. This study represents the first functional analyses of protein network changing during CTEPH, using patient-derived cells. The advanced analytical approach in combination with the study of dysregulated pathways allowed the identification of several important abnormal regulated functional modules, such as mitochondrial metabolism, cell proliferation signalling, oxo-response and fatty acid metabolism, proving the presence of endothelial dysfunction.

Akt-mTOR pathway has multiple downstream effects, related to different cell and tissue types. In CTEPH and related diseases this pathway is activated by thrombin, inducing an enhance of calcium concentration, cell proliferation and endothelium remodelling ${ }^{16}$. In this study the differentially regulated proteins, such as integrin alpha, nitric oxide synthase and protein phosphatase 2, inhibited cell proliferation, showed by the dysregulation of AKT-mTOR, calcium, integrin and NFAT network modules. To date, mTOR has gained wide interest in its potential therapeutic role in several proliferative diseases such as cancer. The role of mTOR in pulmonary hypertension it is not fully understood. Previous work showed an obvious downregulation of of mTOR in lung tissues from patients with pulmonary arterial hypertension $(\mathrm{PAH})$ which was reverted in specific adenovector, mTOR overexpressing mice ${ }^{17}$. After mTOR rebalance mice showed a marked reduction in right ventricular hypertrophy and a reduction of pulmonary artery remodelling. It is not known whether the same phenomena could be happening in CTEPH patients. Another work showed that smooth muscle cells and/or myofibroblasts isolated from pulmonary endarterectomy specimens presented higher expression levels of PDGF and PDGF receptors known to potently activate the Akt/mTOR pathway ${ }^{18}$. CTEPH cells increased phosphorylation of Akt, which subsequently caused an increase in phosphorylated mTOR and p70S6K and that could be inhibited by rapamycin. The levels of mTOR compared to healthy pulmonary artery smooth muscle cells or endothelial cells without PDGF stimulation are not clear to confirm the basal levels of mTOR in patients with CTEPH vs control 


\begin{tabular}{|c|c|c|c|}
\hline Protein Accession numbers & Protein names & Gene names & $\log 2$ (ratio) \\
\hline O00469 & Procollagen-lysine,2-oxoglutarate 5-dioxygenase 2 & PLOD2 & 2.47 \\
\hline Q5U0D2 & Transgelin & TAGLN & 2.41 \\
\hline A0A0P1J1R0 & Translationally-controlled tumor protein & TPT1 & 2.38 \\
\hline P68133 & Actin, alpha skeletal muscle; & ACTA1 & 2.23 \\
\hline V9HW83 & Retinal dehydrogenase 1 & HEL-S-53e & 1.71 \\
\hline H0YNW5 & Deoxyuridine 5-triphosphate nucleotidohydrolase, mitochondrial & DUT & 1.40 \\
\hline J3QR09 & Ribosomal protein L19; & RPL19 & 1.38 \\
\hline Q5ISS1 & ADP-ribosylation factor 1 & ARF1 & 1.34 \\
\hline P68366 & Tubulin alpha-4A chain & TUBA4A & 1.18 \\
\hline Q9UJC5 & SH3 domain-binding glutamic acid-rich-like protein 2 & SH3BGRL2 & 1.17 \\
\hline A0A024R222 & Phosphoserine aminotransferase & PSAT1 & 1.16 \\
\hline B4DDF4 & Calponin; & CNN2 & 1.13 \\
\hline B1AHB1 & DNA helicase;DNA replication licensing factor MCM5 & MCM5 & 1.11 \\
\hline B3KXZ4 & DNA helicase;DNA replication licensing factor MCM2 & MCM2 & 1.08 \\
\hline Q14566 & DNA replication licensing factor MCM6 & MCM6 & 1.05 \\
\hline Q8WX93 & Palladin & PALLD & 1.05 \\
\hline O95433 & Activator of $90 \mathrm{kDa}$ heat shock protein ATPase homolog 1 & AHSA1 & 1.05 \\
\hline K7ELC2 & 40 S ribosomal protein $\mathrm{S} 15$ & RPS15 & 1.02 \\
\hline Q8NG19 & Collagen alpha-1(XVIII) chain;Endostatin & COL18A1 & -1.00 \\
\hline L7UUZ7 & Integrin beta & ITGB3 & -1.01 \\
\hline A0A0S2Z3L0 & Electron transfer flavoprotein subunit alpha, mitochondrial & ETFA & -1.01 \\
\hline Q53YE7 & Amine oxidase [flavin-containing] A & MAOA & -1.02 \\
\hline B3KNK4 & Phosphatidate cytidylyltransferase & CDS2 & -1.03 \\
\hline Q8WYJ5 & Histidine triad nucleotide-binding protein 2, mitochondrial & HINT2 & -1.03 \\
\hline B4E2S7 & Lysosome-associated membrane glycoprotein 2 & LAMP2 & -1.04 \\
\hline A0A024R8T3 & Acyl-coenzyme A oxidase; & ACOX1 & -1.04 \\
\hline B4DEH0 & 39 S ribosomal protein $\mathrm{L} 10$, mitochondrial & MRPL10 & -1.05 \\
\hline A0A0S2Z5A5 & Septin-9 & 43,352 & -1.05 \\
\hline Q96N83 & Podocalyxin & PODXL & -1.06 \\
\hline B4DKM6 & Serum paraoxonase/arylesterase 2 & PON2 & -1.06 \\
\hline A0A024RC61 & Aminopeptidase $\mathrm{N}$ & ANPEP & -1.07 \\
\hline P38117 & Electron transfer flavoprotein subunit beta & ETFB & -1.07 \\
\hline A0A096LPI6 & ES1 protein homolog, mitochondrial & C21orf33 & -1.09 \\
\hline Q6DHZ2 & cAMP-dependent protein kinase type II-beta regulatory subunit & PRKAR2B & -1.09 \\
\hline A0A024R9D7 & 2,4-dienoyl-CoA reductase, mitochondrial & DECR1 & -1.09 \\
\hline P49006 & MARCKS-related protein & MARCKSL1 & -1.10 \\
\hline Q53FJ5 & Prosaposin & PSAP & -1.10 \\
\hline B4E324 & Carboxypeptidase; & CTSA & -1.10 \\
\hline Q9NV96 & Cell cycle control protein $50 \mathrm{~A}$ & TMEM30A & -1.10 \\
\hline Q14XT3 & Cytochrome c oxidase subunit 2 & COX2 & -1.11 \\
\hline P35580 & Myosin-10 & MYH10 & -1.11 \\
\hline B7Z8A2 & Prenylcysteine oxidase 1 & PCYOX1 & -1.11 \\
\hline Q96CM8 & Acyl-CoA synthetase family member 2 , mitochondrial & ACSF2 & -1.12 \\
\hline Q9Y4D7 & Plexin-D1 & PLXND1 & -1.15 \\
\hline A0A024RAB6 & $\begin{array}{l}\text { Basement membrane-specific heparan sulfate proteoglycan core } \\
\text { protein;Endorepellin;LG3 peptide }\end{array}$ & HSPG2 & -1.16 \\
\hline B2R7D2 & Multiple inositol polyphosphate phosphatase 1 & MINPP1 & -1.16 \\
\hline A0A1B0GW44 & Cathepsin D; & HEL-S-130P & -1.17 \\
\hline A0A024RAD8 & Delta-1-pyrroline-5-carboxylate dehydrogenase, mitochondrial & ALDH4A1 & -1.17 \\
\hline P16284 & Platelet endothelial cell adhesion molecule & PECAM1 & -1.18 \\
\hline Q9UHL4 & Dipeptidyl peptidase 2 & DPP7 & -1.18 \\
\hline Q8TDB4 & Protein MGARP & MGARP & -1.19 \\
\hline P51970 & NADH dehydrogenase [ubiquinone] 1 alpha subcomplex subunit 8 & NDUFA8 & -1.21 \\
\hline Q9BVJ8 & Beta-hexosaminidase;Beta-hexosaminidase subunit alpha & HEXA & -1.21 \\
\hline B4DJQ8 & Dipeptidyl peptidase 1; & CTSC & -1.23 \\
\hline A8K9J6 & CD276 antigen & CD276 & -1.23 \\
\hline Q53FB6 & Aldehyde dehydrogenase, mitochondrial & ALDH2 & -1.25 \\
\hline
\end{tabular}




\begin{tabular}{|c|c|c|c|}
\hline Protein Accession numbers & Protein names & Gene names & $\log 2$ (ratio) \\
\hline O75923 & Dysferlin & DYSF & -1.25 \\
\hline P04275 & von Willebrand factor;von Willebrand antigen 2 & VWF & -1.26 \\
\hline P49407 & Beta-arrestin-1 & ARRB1 & -1.27 \\
\hline Q9Y2Q5 & Ragulator complex protein LAMTOR2 & LAMTOR2 & -1.28 \\
\hline Q6IB89 & NADH dehydrogenase [ubiquinone] 1 alpha subcomplex subunit 7 & NDUFA7 & -1.29 \\
\hline Q9NSC5 & Homer protein homolog 3 & HOMER3 & -1.31 \\
\hline Q53H18 & Beta-galactosidase & GLB1 & -1.32 \\
\hline A0A0C4DFN8 & NADPH:adrenodoxin oxidoreductase, mitochondrial & FDXR & -1.34 \\
\hline A8K335 & Gamma-glutamyl hydrolase & GGH & -1.34 \\
\hline A0A140VJL0 & 3-hydroxyisobutyryl-CoA hydrolase, mitochondrial & HIBCH & -1.35 \\
\hline A0A024R8Q1 & Lysosomal alpha-glucosidase; & GAA & -1.36 \\
\hline B3KQQ0 & Peptidyl-prolyl cis-trans isomerase FKBP9 & FKBP9 & -1.39 \\
\hline A0A090N7X0 & GTPase IMAP family member 4 & HIMAP4 & -1.39 \\
\hline Q5URX0 & Beta-hexosaminidase; & HEXB & -1.41 \\
\hline P36551 & Oxygen-dependent coproporphyrinogen-III oxidase, mitochondrial & CPOX & -1.41 \\
\hline A0A024RA75 & 3-hydroxyisobutyrate dehydrogenase & HIBADH & -1.42 \\
\hline B4DTT0 & $\mathrm{N}$-acetylglucosamine-6-sulfatase & DKFZp686E12166 & -1.44 \\
\hline A0A090N8H2 & GTPase IMAP family member 8 & hIAN6 & -1.45 \\
\hline Q7Z7M4 & Superoxide dismutase; & SOD2 & -1.47 \\
\hline Q92626 & Peroxidasin homolog & PXDN & -1.48 \\
\hline Q14165 & Malectin & MLEC & -1.52 \\
\hline B4DSE2 & Tripeptidyl-peptidase 1 & TPP1 & -1.53 \\
\hline P04040 & Catalase & CAT & -1.55 \\
\hline Q96DI8 & Heme oxygenase 1 & HMOX1 & -1.55 \\
\hline B4DJI2 & Granulins; & GRN & -1.56 \\
\hline Q5TBU5 & Adipogenesis regulatory factor & hCG_1773630 & -1.58 \\
\hline P30837 & Aldehyde dehydrogenase $\mathrm{X}$, mitochondrial & ALDH1B1 & -1.58 \\
\hline Q5NKV8 & Intercellular adhesion molecule 1 & ICAM1 & -1.59 \\
\hline A0A024R5L0 & Lysosomal Pro-X carboxypeptidase & PRCP & -1.91 \\
\hline Q8NHP8 & Putative phospholipase B-like 2; & PLBD2 & -2.01 \\
\hline A0A090N8Z4 & GTPase IMAP family member 1 & IMAP1 & -2.04 \\
\hline K7ERP4 & Glutathione peroxidase; & GPX4 & -2.18 \\
\hline A0A024RDG9 & 3-hydroxybutyrate dehydrogenase type 2 & BDH2 & -2.21 \\
\hline B2R769 & Endoplasmic reticulum aminopeptidase 2 & ERAP2 & -2.53 \\
\hline V9HW74 & Ubiquitin carboxyl-terminal hydrolase; & HEL-117 & -3.17 \\
\hline
\end{tabular}

Table 1. List of protein differentially regulated in CTEPH patients versus controls.

cells. Additionally, Ogawa et al. work significantly differs with the present findings as the cell population isolated from the pulmonary endarterectomy specimens is different ${ }^{18}$. In the present study only endothelial cells and not smooth muscle cells or myofibroblasts were isolated. Despite both cell lines co-exist in pulmonary arteries and interact constantly between each other both cells play different roles in vascular homeostasis. This could explain why the expression levels of mTOR in endothelial CTEPH cells are different to smooth muscle cells. However, this protein functional module can be targeted by drug or an indication for early diagnosis of CTEPH disease since its activation allows the remodelling of vascular endothelium.

The quantitative proteomics approach showed for the first time the protein PLOD2 as the most upregulated protein attesting its key role in CTEPH. PLOD2 is an attachment site for carbohydrates and it is involved in the stability of collagen fibrils. Collagen plays a key role in the maintenance of integrity and elasticity of normal vessel walls as well as atherosclerotic vessel walls and it stimulates the thrombosis formation and scar formation processes particularly in PAH patients ${ }^{19}$. Under the hypoxic conditions, PLOD2 mediate the remodelling of extracellular matrix in synergy with collagen prolyl hydroxylases (P4HA1 and P4HA2) ${ }^{20}$. PLOD2 is involved in collagen deposition that induces the reduction of vascular elasticity ${ }^{20}$. PLOD2 promotes the cross-link of collagen, important event for the formation and stabilization of insoluble collagen fibrils. Collagen containing pyridinoline cross-links are difficult to degrade by collagenases, increasing collagen deposition and tissue stiffness. Over-expression of PLOD2 and over-production of insoluble collagen fibres in CTEPH disease could increase the collagen 's deposition, tissue's stiffness, myofibroblast's differentiation and platelet's aggregation ${ }^{13,14}$. COL18A1, involved in the maintenance of vessel wall integrity and structure in atherogenesis ${ }^{20,21}$, was down regulated. On the other hand, TAGLN showed an extensive upregulation with a ratio of 5.3 in CTEPH-ECs. Recent study has shown that TAGLN overexpression promoted the proliferation and migration of human pulmonary 
Cell movement 1

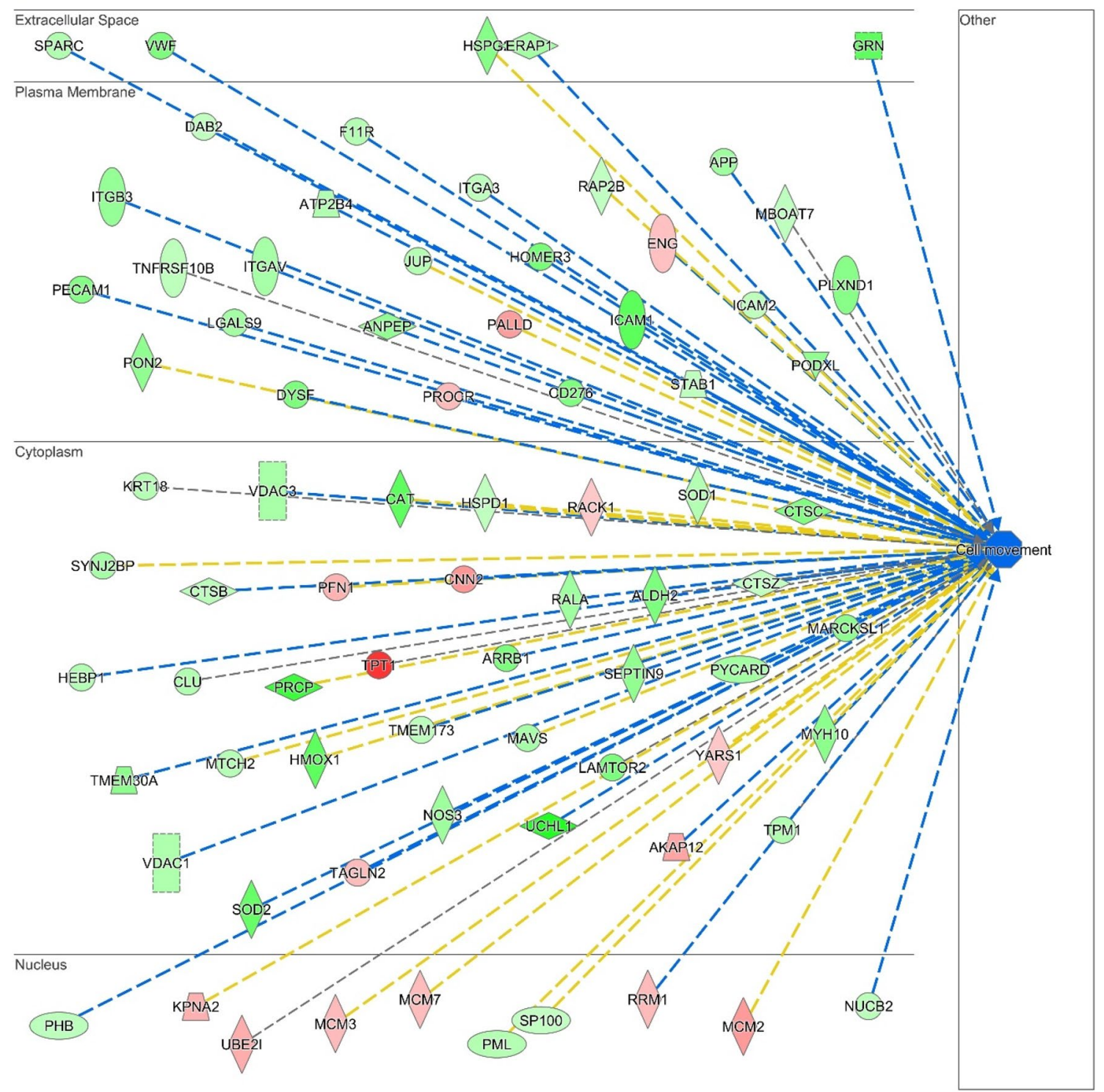

Figure 2. Molecules involved in cell movement significantly altered in CTEPH patients (last version IPA, QIAGEN Inc., https://www.qiagenbioinformatics.com/products/ingenuitypathway-analysis), in red the increased genes, in green those decreased. The color intensity is positive related to the up- or down-gene's regulation; orange line leads to activation, yellow lines for findings inconsistent with state of downstream molecule; grey line for effect not predicted.

arterial smooth muscle cells (PASMC), strengthened cytoskeleton; suppression of this protein activated PASMC apoptosis, reducing cell proliferation and migration ${ }^{22}$. The higher expression of TAGLN might be related to the hyperproliferative phenotype of CTEPH-ECs and it might have an important role in endothelial dysfunction. Furthermore, a drastic alteration in mitochondrial respiratory chain complexes was described by network analyses.

Haemolysis and oxidative stress are peculiar features in pulmonary hypertension and endothelial dysfunction ${ }^{23-27}$. Glutathione, a major non-protein thiol in living organisms, plays a central role in coordinating the body's antioxidant defence processes ${ }^{28-30}$. Perturbation of glutathione status of a biological system has been reported to lead to serious consequences. In the present study, low level of GSH in CTEPH dysfunctional ECs was observed. Interestingly quantitative proteomics analysis showed a down-regulation of GPX4 and GPX1 proteins that catalyse the formation of glutathione disulphide in presence of hydrogen peroxide (Table 1). GPX1 decreased the bioavailability of $\mathrm{NO}$ and increased the oxidative stress ${ }^{31}$. Therefore, the underlying mechanisms might be attributed to the unavailability of GSH, the substrate for GPX. The capacity of the glutathione system to deal with free radicals mainly depends upon the activity of peroxidase and glutathione reductase (GR). Glutathione reductase keeps a high ratio of GSH/GSSG in normal cells. In CTEPH-ECs glutathione reductase might be in lower levels, which in turn is linked to the decreased concentration of GPx and reduced glutathione. The down regulation of identified GPX4, GPX1, NOS3 proteins attested the increment of oxidative stress. Among 


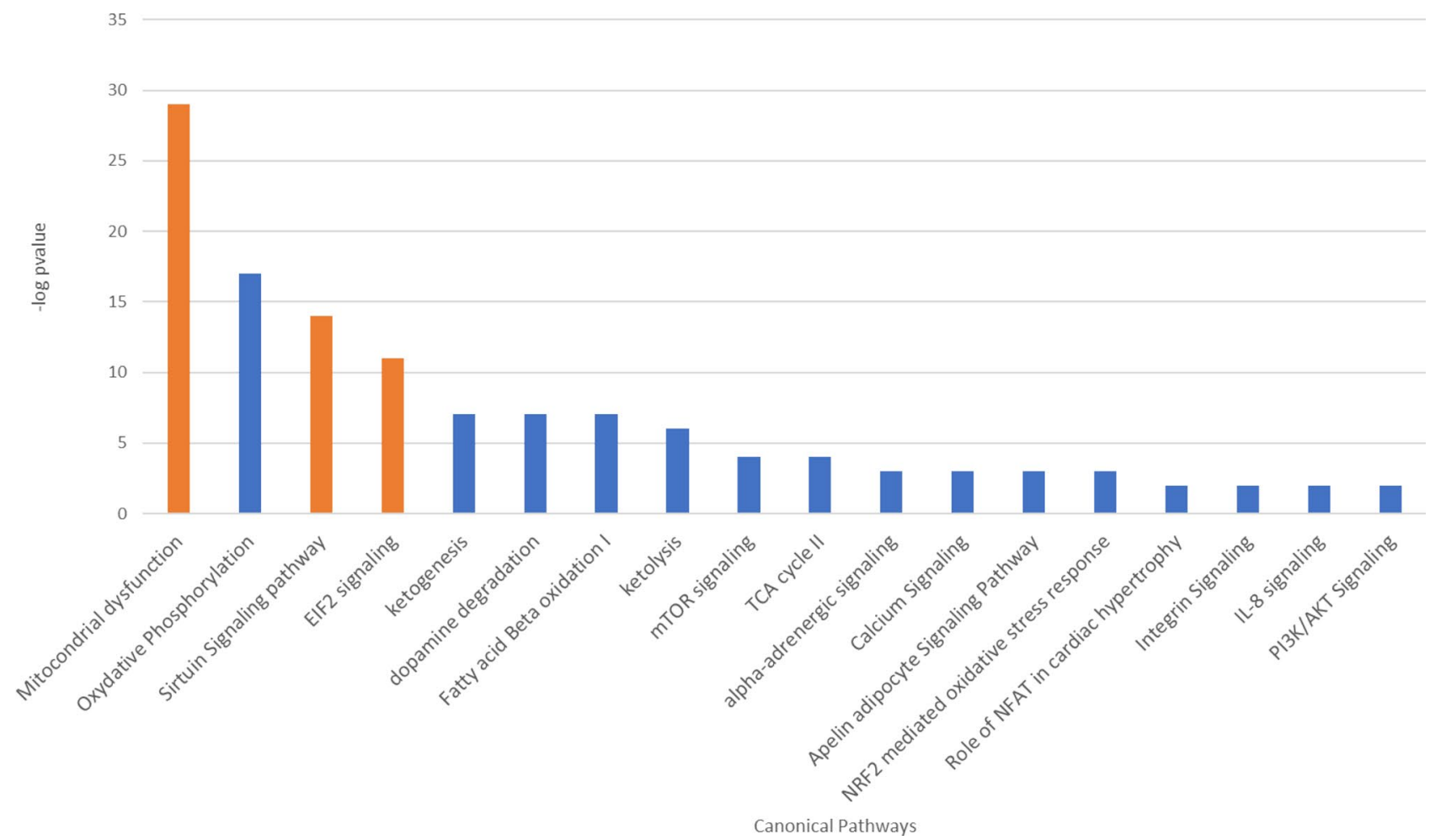

Figure 3. Canonical Pathways that are differentially regulated in $\mathrm{CTEPH}$ patients versus controls, in orange the up regulated $(\mathrm{z}$ score $>0$ ) and in blue the down regulated ones (zscore $<0$ ) (last version IPA, QIAGEN Inc., https ://www.qiagenbioinformatics.com/products/ingenuitypathway-analysis).

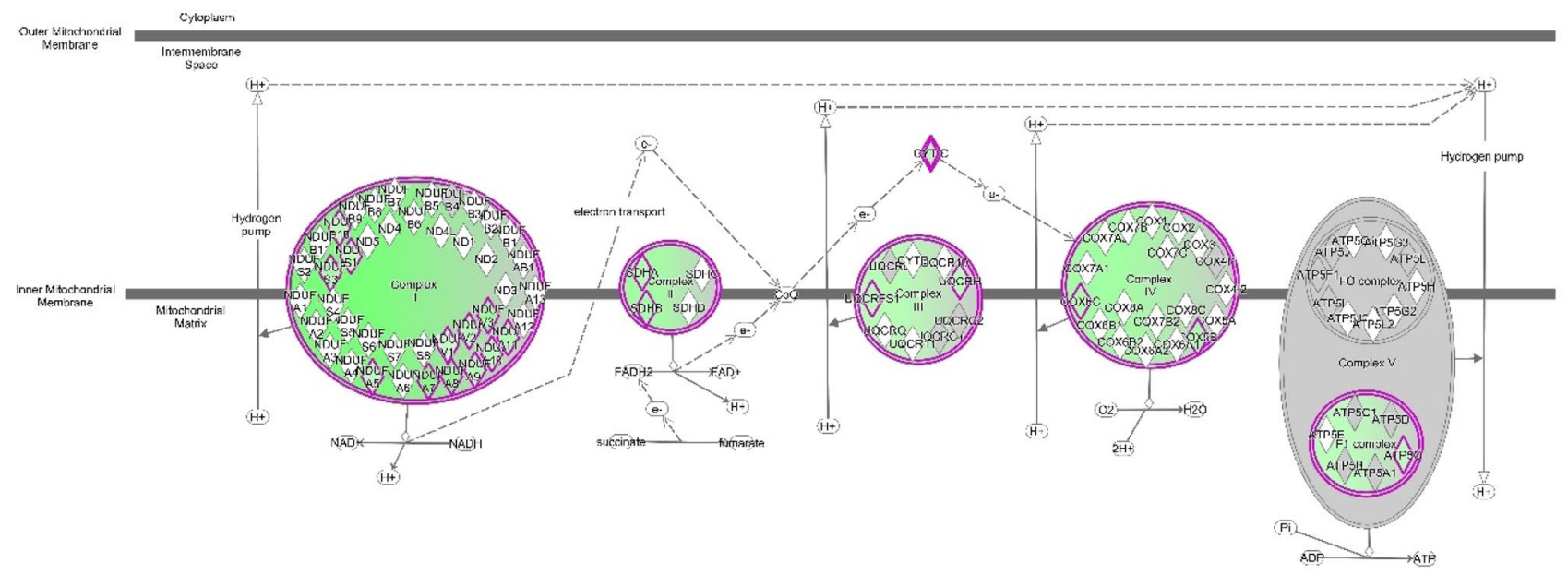

Figure 4. Pathways involved in mitochondrial dysfunction significantly altered in CTEPH patients ((last version IPA, QIAGEN Inc., https://www.qiagenbioinformatics.com/products/ingenuitypathway-analysis), in red the increased genes, in green those decreased. The color intensity is positive related to the up- or downgene's regulation; orange line leads to activation, yellow lines for findings inconsistent with state of downstream molecule; grey line for effect not predicted.

these GPX4 found as one of the most down-regulated protein. Under physiological conditions, the antioxidants related to glutathione peroxidase family proteins GPX1 and GPX4 protects the cells from oxidative stress ${ }^{32}$. GPX 4 prevents the cells from ferroptosis, a non-apoptotic cell death as consequence of iron-dependent accumulation of lipid reactive oxygen species (ROS) ${ }^{33}$. Elevated levels of GPX1 protected the cultured HPAECs from hydrogen peroxide induced cell death ${ }^{34}$. Reduced expression of GPX1 can also lead to inflammatory activation of ECs, that further increase to a proatherogenic endothelial phenotype ${ }^{35}$. Genetic deletion of GPX1 decreased bioavailability of NO and increased the oxidative stress ${ }^{35}$. Moreover, normal ECs always generate endothelial nitric oxide synthase (eNOS), which is essential to maintain various endothelial functions including the vascular smooth 


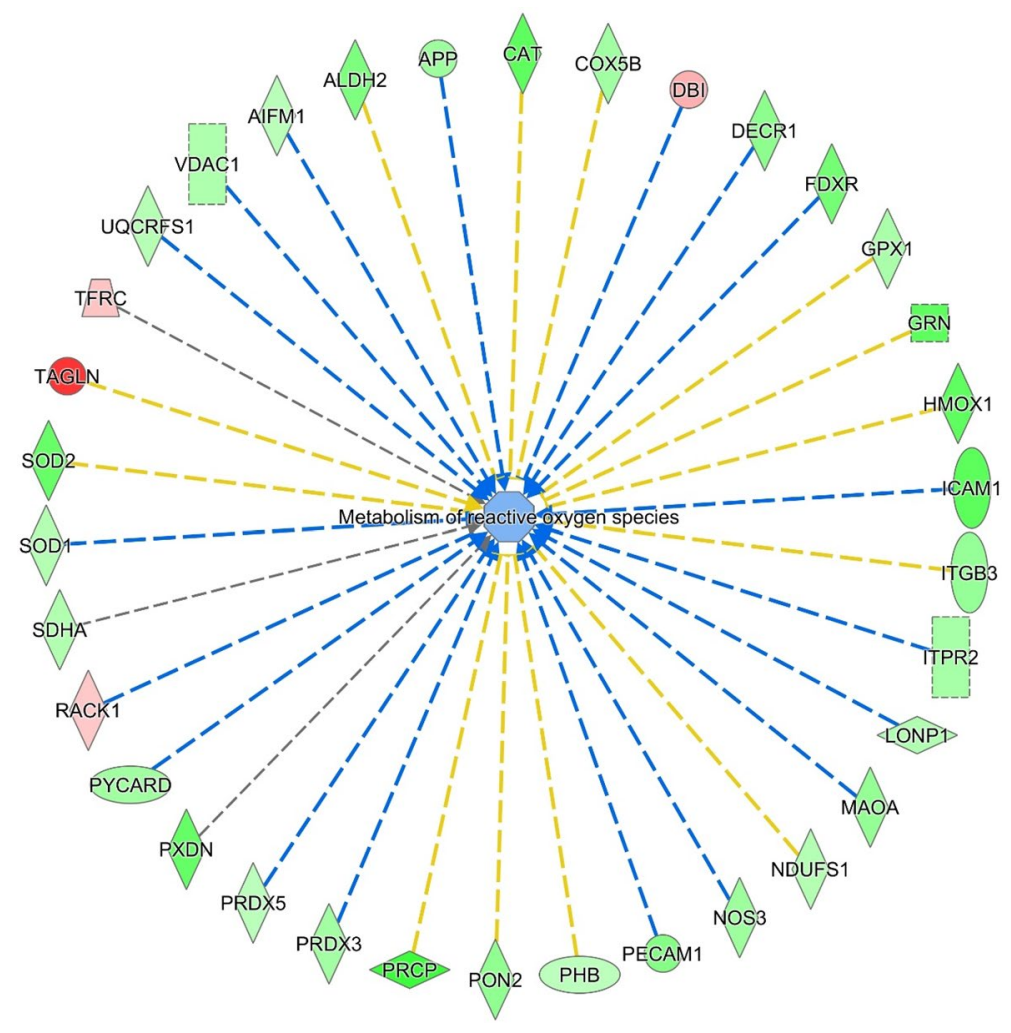

Figure 5. Molecules involved in Metabolism of reactive oxygen species significantly altered in CTEPH patients (IPA), in red the increased genes, in green those decreased.

\section{Advanced Oxidation Protein Products (AOPP)}

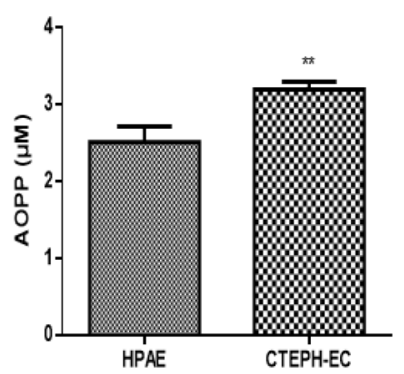

Total protein carbonyl content (PCO)

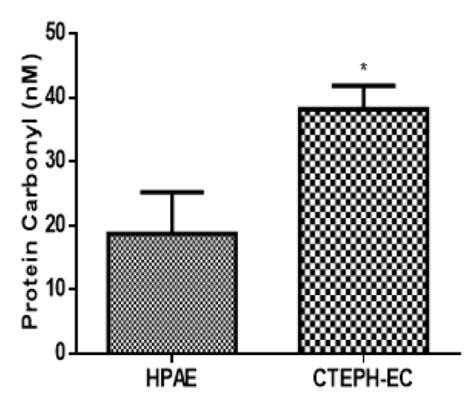

Intracellular ROS

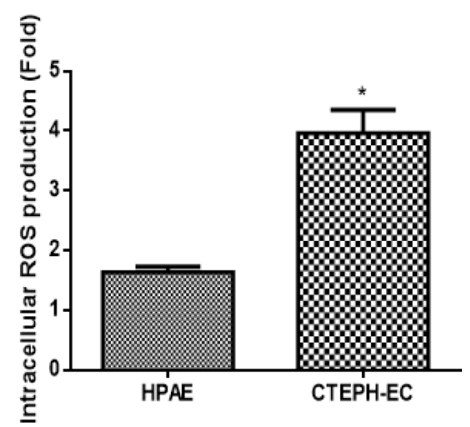

Levels of GSH/GSSG ratio

Levels of NADPH/NADP ratio
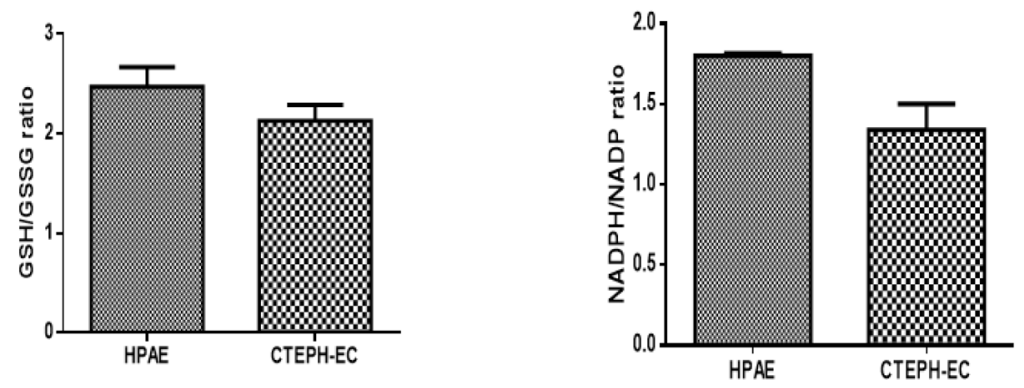

Figure 6. Levels of oxidants and antioxidants in dysfunctional ECs of CTEPH. Data were shown in mean \pm SEM. Differences were considered significant when $\mathrm{p}<0.05\left(^{*}\right), 0.001<\mathrm{p}<0.01\left({ }^{* *}\right), \mathrm{p}<0.001\left(^{* * *}\right)$, $\mathrm{p}<0.0001\left({ }^{* * *}\right)$. 
muscle relaxation, vascular endothelial growth factor (VEGF) induced angiogenesis, suppression of inflammatory cell migration and adhesion, angiogenesis, inhibition of thrombosis and coagulation. The impairment of eNOS causes the endothelial dysfunction and oxidative stress which further increases the atherogenesis. The endothelial NOS3 protein, responsible to produce NO, was found down regulated and vascular endothelium glycoprotein ENG, involved in the negative regulation of NO production, was found up regulated. Therefore, the reduction in NO bioavailability, resulting from reduced NO production and/or increased NO degradation by superoxide anion, describes the onset of endothelial dysfunction ${ }^{27}$.

Moreover, NADPH oxidase-derived ROS play a role in vascular pathology as well as in the maintenance of normal physiological vascular function ${ }^{36}$. NADPH has been suggested to act as an indirectly operating antioxidant, thus maintaining the antioxidative power of glutathione ${ }^{37}$. In the present study, lower level of NADPH was observed in CTEPH, suggesting the increasing of oxidative stress in dysfunctional ECs.

The increased levels of AOPPs might explain the involvement of higher global inflammatory activity in endothelial dysfunction ${ }^{38}$. Increased AOPPs production might be involved in the generation of various types of $\operatorname{ROS}^{39}$. The accumulation of ROS has been well established in the pathogenesis of several diseases including diabetes $^{40}$, atherosclerosis ${ }^{41}$, renal ischemia ${ }^{42}$, inflammatory diseases ${ }^{43}$ and cancer ${ }^{44}$. Increased production of ROS in dysfunctional ECs might enhance pro-inflammatory pathways and affect protein, lipid and enzymatic activity.

In summary, the mechanisms underlying endothelial dysfunction implicates the alterations in metabolism, inflammation and oxidative stress events.

\section{Conclusions}

This is the first study to attempt the description of molecular network in in endothelial dysfunction associated with CTEPH using patient-derived pathological ECs. Quantitative proteomic profiling in endothelial dysfunction reveals the differentially regulated proteins that might behave as a biomarker signature of vascular injury, metabolism and oxidative stress. Increased production of oxidants and decreased production of antioxidant biomarkers as well as down regulation of protein which had an antioxidant property strongly explained the correlation of the role of oxidative stress in endothelial dysfunction of CTEPH.

In conclusion the study unveiled the pathophysiological mechanisms and some important functional modules, constituted by differentially regulated proteins, dysregulated during the disease.

Potential limitations and future perspective of the study. Although we used a statistically significant number of patients-derived ECs to identify underlying mechanisms associated with endothelial dysfunction of CTEPH, further experiments need to be performed to validate the expression of proteins using clinical samples e.g. blood, for determining them as a potential prognostic marker for early prediction of endothelial dysfunction. Despite knowing the background of patients from whom we have collected specimens to isolate ECs, the long-term medical history and potential risk factors to which they might have exposed is anonymous. The perspective of the current study would be to perform more experiments to unveil mechanistic and functional characteristics of PLOD2 and ENG1, which might provide an in-depth understanding of endothelial dysfunction. To understand the effect of these dysregulated proteins in downstream signaling pathways, gene overexpression and knockdown analysis will be performed in vitro. Additionally, to understand whether the upregulation of PLOD2 and ENG1 might play a key role in inducing oxidative stress, we will use various biochemical and molecular biology approaches followed by gene overexpression in ECs. To further overcome the limitations of the current study, perspective studies would be performed using patient-specific induced pluripotent stem cell-derived endothelial cells (iPSC-ECs).

\section{Materials and methods}

Endothelial cell culture. Commercially available human pulmonary artery endothelial cells (HPAE) were used as controls. Five individual ECs lines, derived from surgical specimens obtained in patients with CTEPH (CTEPH-ECs) who underwent pulmonary endarterectomy, were received from Dr. Olga Tura, Research Scientist, Department of Pulmonary Medicine, Hospital Clínic-Institut d'Investigacions Biomèdiques August Pi iSunyer (IDIBAPS), University of Barcelona, Spain. The study was conducted in accordance with the Declaration of Helsinki, was approved by the Committee on Human Research of our institution and all subjects gave written informed consent (Comité Ético de Investigación Clínica del Hospital Clínic de Barcelona). The clinical characteristics of CTEPH endothelial cells are summarized in Supplementary Table 4.

The specimens containing thromboembolic material with clear thrombus were obtained after pulmonary endarterectomy (PEA) surgery from CTEPH patients at IDIBAPS. Material were washed with PBS and minced into 1-2 mm pieces. Explants were seeded into 0.2\% gelatin-coated 6-well plates and maintained under standard cell culture conditions. After $24 \mathrm{~h}$, tissue explants, non-adherent cells, and debris were aspirated. Medium was changed every other day until the first passage of pulmonary endothelial outgrowth cells emerged and cells were cultured as previously described ${ }^{45}$.

Protein extraction and In-solution trypsin digestion. Confluent cells were trypsinized and collected by centrifugation at $1200 \mathrm{rpm}$ for $5 \mathrm{~min}$. Harvested cells were washed two times with cold PBS. The cell pellets were resuspended in the solubilization buffer $(8 \mathrm{M}$ urea in $50 \mathrm{mM}$ Tris- $\mathrm{HCl}, 30 \mathrm{mM} \mathrm{NaCl}, \mathrm{pH} 8.5$ and $1 \%$ protease inhibitor) and incubated on ice for 5-10 min. Cell lysates were further homogenized by sonication in an ice bath for three times each for $5 \mathrm{~s}$ with $30 \mathrm{~s}$ intervals, using an ultra sonicator. Samples were centrifuged at $14,000 \mathrm{rpm}$ for $20 \mathrm{~min}$ at $4^{\circ} \mathrm{C}$. The protein supernatant was collected into the new Eppendorf tube and pelleted cell debris was discarded. Samples were stored at $-80^{\circ} \mathrm{C}$ until we use it for further experiments. The protein estimation was carried out by using the Bradford assay. 
$10 \mu \mathrm{g}$ of total protein was resuspended in $50 \mathrm{mM}$ ammonium bicarbonate. Reduction was carried out by incubating $5 \mathrm{mM}$ final concentration of DTT for $30 \mathrm{~min}$ at $52{ }^{\circ} \mathrm{C}$, followed by an alkylation with $15 \mathrm{mM}$ final concentration of iodoacetamide for $20 \mathrm{~min}$ in the dark at room temperature. Trypsin digestion was allowed at $37^{\circ} \mathrm{C}$ overnight, with an enzyme: substrate ratio of 1:10. The resulting peptides were used for MS analysis.

Mass spectrometry and data analyses. Tryptic peptides were analyzed using a Dionex Ultimate 3000 nano-LC system (Sunnyvale CA, USA) connected to an Orbitrap Fusion Tribrid Mass Spectrometer (Thermo Scientific, Bremen, Germany) equipped with a nano-electrospray ion source. Peptide mixtures were pre-concentrated onto an Acclaim PepMap $100-100 \mu \mathrm{m} \times 2 \mathrm{~cm} \mathrm{C18}$ and separated on EASY-Spray column, $15 \mathrm{~cm} \times 75 \mu \mathrm{m}$ ID packed with Thermo Scientific Acclaim PepMap RSLC C18, $3 \mu \mathrm{m}, 100 \AA$ A. The temperature was setting to $35{ }^{\circ} \mathrm{C}$ and the flow rate was $300 \mathrm{~nL} / \mathrm{min}$. Mobile phases were the following: $0.1 \%$ Formic acid (FA) in water (solvent A); $0.1 \%$ FA in water/acetonitrile (solvent B) with $2 / 8$ ratio. Peptides were eluted from the column with the following gradient: $4 \%$ to $28 \%$ of B for $90 \mathrm{~min}$ and then $28 \%$ to $40 \%$ of B in $10 \mathrm{~min}$, and to $95 \%$ within the following $6 \mathrm{~min}$ to rinse the column. Column was re-equilibrated for $20 \mathrm{~min}$. Total run time was $130 \mathrm{~min}$. One blank was run between triplicates to prevent sample carryover. MS spectra were collected over an $\mathrm{m} / \mathrm{z}$ range of 375-1500 at 120,000 resolutions, operating in the data dependent mode, cycle time of 3 s. Higher-energy collisional dissociation (HCD) was performed with collision energy set at 35\%. Each sample was analysed in three technical triplicates ${ }^{45}$.

Resulting MS raw files from all the technical and biological replicates were analysed by using MaxQuant software $^{48}$ (version 1.6.2.3). Andromeda search engine was used to identify MS/MS based peptide and proteins in MaxQuant comprises a target-decoy approach with less than $1 \%$ of False Discovery Rate (FDR). In the present study we used Uniprot_Homosapiens database. Trypsin was used for enzyme specificity, 2 missed cleavages and maximum five number of modifications per peptide was allowed. Methionine oxidation and acetylation ( $\mathrm{N}$ terminus) was used as a variable modification. Carbamidomethylation was used as a fixed modification. The proteins were selected with a minimum of two peptides. For the label-free quantification of proteins, we applied MaxLFQ algorithm. Match between the runs option was enabled and remaining default parameters were permitted. Data available on request from the authors.

An open source Perseus software ${ }^{46}$ (version 1.6.1.3; Max Planck Institute of Biochemistry, Germany) was used for the identification of statistically significantly differentially regulated proteins. The interpretation and visualization of results obtained from MaxQuant software were performed by a two-sample t-test using Perseus (v1.6.1.3, Max Planck Institute of Biochemistry, Germany). Statistical parameters ( $p<0.05 ; \mathrm{q}<0.05, \mathrm{q}=$ FDR adjusted p-value) were set to identify the differentially expressed proteins between samples, categorized in two groups: CTEPH-EC (patients) and HPAEC (controls) (log2 ratio). Variabilities of biological replicates were measured with Pearson correlation coefficient values of the LFQ intensities. The differentially regulated proteins with a minimum of two peptides and FDR adjusted $\mathrm{p}$-value $<0.05$ were considered as statistically significant ${ }^{46}$.

Protein network analysis. The network protein analysis related to significantly altered proteins was carried out by IngenuityPathwaysAnalysis(IPA)(QIAGENInc., https://www.qiagenbioinformatics.com/products/ ingenuitypathway-analysis). The statistical enrichment of involved pathways is performed by the right-tailed Fisher's exact test, in correlation with QIAGEN Knowledge Base, assigning a p-value. The core analyses performed by IPA, using the differentially expressed proteins in the uploaded dataset, assess signaling pathways, molecular interaction network and biological functions that are likely to be perturbed. The overall activation/ inhibition states of canonical pathways are predicted based on a $\mathrm{Z}$-score algorithm. This $\mathrm{Z}$-score is used to statistically compare the uploaded dataset with the pathway patterns. The pathways are colored to indicate their activation z-scores: orange predict a gain of function, while blue a loss of function. The pathway is activated when molecules' causal relationships with each other (i.e., activation edge and the inhibition edge between the molecules based on literature findings) generate an activity pattern for the molecules and the end-point functions in the pathway.

Quantitative gene expression analysis. Total RNA extraction and conversion to first-strand cDNA were done using iScript RT-qPCR sample preparation reagent (Bio-Rad) and Maxima First Strand cDNA Synthesis Kit (Thermo Scientific), respectively according to the manufacturer's protocol. Quantitative RT-PCR analysis was performed using an ABI PRISM 7000 Sequence detection system cDNA was amplified in triplicate using Maxima SYBR Green/ROX qPCR Master Mix (Thermo Scientific). The thermal cycling programme started at $95^{\circ} \mathrm{C}$ for $10 \mathrm{~min}$ for enzyme activation, followed by 40 cycles of amplification at $95^{\circ} \mathrm{C}$ for $15 \mathrm{~s}$, followed by $60^{\circ} \mathrm{C}$ for $1 \mathrm{~min}$. Gene expression was normalized to the housekeeping gene RPLP0. Relative expression of targeted genes was determined using the $2^{-\Delta \Delta C T}$ method. P value below 0.05 were considered as statistically significant.

Biomolecular assays. Advanced oxidation protein product (AOPP) levels were analysed by using the OxiSelect AOPP kit (STA318, Cell Biolabs, San Diego, CA, USA) following the manufacturer's protocol. Briefly, the confluent cells were collected, lysed in buffer $(150 \mathrm{mM} \mathrm{NaCl}, 1 \% \mathrm{NP}-40,0.5 \%$ sodium deoxycholate, $0.1 \%$

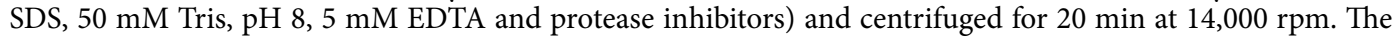
pellet containing cell debris was discarded; the protein supernatant was collected and quantified by Bradford assay. $50 \mu \mathrm{g}$ of protein in $200 \mu \mathrm{L}$ final volume of control and pathological cell lysates were subjected to $10 \mu \mathrm{L}$ of chloramine reaction initiator followed by $20 \mu \mathrm{L}$ of stop solution. Absorbance was recorded at $340 \mathrm{~nm}$ by using the spectrophotometric plate reader (PowerWave biotek). Cell lysates used in this assay was prepared from the ECs in passages between 6-9. Experiments were performed with biological and technical replicates ${ }^{47}$. 
Protein carbonyl content, a general indicator of protein oxidation was measured fluorometrically $(480 \mathrm{~nm}$ excitation/530 nm emission) using a commercial kit (Cell Biolabs, OxiSelect No. STA-307) as per the instructions provided by the manufacturer. Experiments were performed with biological and technical replicates.

ROS was measured by OxiSelect ROS Assay Kit (Cell Biolabs, STA-342) as per the manufacturer protocol. Briefly, the control and pathological ECs were grown in 96 well plate at a density of $9 \times 10^{3}$ cells and incubated with the dichloro-dihydro-fluorescein diacetate (DCFH-DA) containing medium at $37^{\circ} \mathrm{C}$ for 60 min in the dark. The confluent cells were lysed, and the fluorescence was recorded by using the fluorometric plate reader at $480 \mathrm{~nm} / 530 \mathrm{~nm}$. Experiments were performed with biological and technical replicates.

GSH/GSSG ratio in control and pathological EC cultures was determined using GSH/GSSG Ratio Detection Assay Kit (Fluorometric-Green) (Abcam Inc. \#ab138881) according to the manufacturer's guidelines. The samples were prepared by lysis of total cell protein in 0.5\% NP-40 lysis buffer followed by a dilution of 1:50 for GSH analysis. In brief, serial dilution of GSH and GSSG stock standards were prepared along with assay mixtures for detection of GSH and total GSH using $100 \times$ Thiol green stock solutions, assay buffer and GSSG probe. A one- step fluorometric reaction of sample with respective assay buffers were incubated for $30 \mathrm{~min}$. Fluorescence intensity was then monitored at Ex/Em of 490/520 nm. GSSG was determined by subtracting GSH from total GSH. Finally, the ratio of GSH was plotted against GSSG to obtain the GSH activity ${ }^{48}$. Experiments were performed with biological and technical replicates.

NADPH/NADP ratio in control and pathological ECs was determined by using NADP/NADPH quantification kit (Sigma) as per the manufacturer's protocols. Briefly, $1 \times 10^{6}$ confluent cells were pelleted and washed with cold PBS. Cells were extracted with $800 \mu \mathrm{l}$ of NADP/NADPH extraction buffer and allowed to incubation for $10 \mathrm{~min}$ on ice. Samples were centrifuged at 10,000 $\mathrm{g}$ for $10 \mathrm{~min}$ and supernatant used for determining the ratio of NADP and NADPH. To remove the enzymes that may consume NADPH rapidly were removed by filtering through a $10 \mathrm{kDA}$ cut-off spin filter (Millipore, UFC501096). An aliquot of the supernatant was heated at $60^{\circ} \mathrm{C}$ for $30 \mathrm{~min}$ to decompose the $\mathrm{NADP}^{+}$, cooled on ice, and spun quickly to remove the precipitate. Another aliquot of the supernatant was not heated. Both aliquots were reacted with $\mathrm{NADP}^{+}$cycling buffer and enzyme mix (containing glucose-6-phosphate dehydrogenase $(\mathrm{G} 6 \mathrm{PDH})$ for $5 \mathrm{~min}$ at room temperature to convert $\mathrm{NADP}^{+}$to NADPH. The solutions were then incubated with NADPH developer for $2 \mathrm{~h}$ and the absorbance measured at $450 \mathrm{~nm}$. The amount of NADPH (heated sample) and the total $\mathrm{NADP}^{+}$and NADPH (unheated sample) were quantified from an NADPH standard curve ${ }^{49}$. Experiments were performed with biological and technical replicates.

Received: 26 June 2020; Accepted: 18 February 2021

Published online: 10 March 2021

\section{References}

1. Kim, N. H. et al. Chronic thromboembolic pulmonary hypertension. Eur. Respir. J. 24, 1801915 (2019).

2. Wynants, M. et al. Effects of C-reactive protein on human pulmonary vascular cells in chronic thromboembolic pulmonary hypertension. Eur. Respir. J. 40, 886-894 (2012).

3. Bonderman, D. et al. Role for staphylococci in misguided thrombus resolution of chronic thromboembolic pulmonary hypertension. Arterioscler. Thromb. Vasc. Biol. 28, 678-684 (2008).

4. Albani, S., Biondi, F., Stolfo, D., Lo Giudice, F. \& Sinagra, G. Chronic Thromboembolic Pulmonary Hypertension (CTEPH). J. Cardiovasc. Med. 20, 159-168 (2019).

5. Dorfmüller, P. et al. Microvascular disease in chronic thromboembolic pulmonary hypertension: a role for pulmonary veins and systemic vasculature. Eur. Respir. J. 44, 1275-1288 (2014).

6. Lum, H. \& Roebuck, K. A. Oxidant stress and endothelial cell dysfunction. Am. J. Physiol. Physiol. 280, C719-C741 (2001).

7. Ranchoux, B., et al. Endothelial dysfunction in pulmonary arterial hypertension: an evolving landscape (2017 Grover Conference Series). Pulmonary Circulation. SAGE Publications Ltd January 1 (2018).

8. Makino, A., Firth, A. L. \& Yuan, J. X. J. Endothelial and smooth muscle cell ion channels in pulmonary vasoconstriction and vascular remodeling. Compr. Physiol. 1, 1555-1602 (2011).

9. Hoeper, M. M. et al. Chronic thromboembolic pulmonary hypertension. Lancet. Respir. Med. 2, 573-582 (2014).

10. Kim, N. H. et al. Chronic thromboembolic pulmonary hypertension. J. Am. Coll. Cardiol. 62, D92-D99 (2013).

11. Konstantinides, S. et al. 2014 ESC guidelines on the diagnosis and management of acute pulmonary embolism. Kardiol. Pol. 72, 997-1053 (2014).

12. Zhang, Y. et al. Advances in targeted therapy for chronic thromboembolic pulmonary hypertension. Heart Fail. Rev. 24, 949-965 (2019).

13. Mia, M. M. \& Bank, R. A. The pro-fibrotic properties of transforming growth factor on human fibroblasts are counteracted by caffeic acid by inhibiting myofibroblast formation and collagen synthesis. Cell Tissue Res. 363, 775-789 (2016).

14. Van Der Slot, A. J. et al. Elevated formation of pyridinoline cross-links by profibrotic cytokines is associated with enhanced lysyl hydroxylase 2b levels. Biochim. Biophys. Acta Mol. Basis Dis. 1741, 95-102 (2005).

15. Ogawa, A. et al. Thrombin-mediated activation of Akt signaling contributes to pulmonary vascular remodeling in pulmonary hypertension. Physiol. Rep. 1(7), e00190 (2013).

16. Simonneau, G. et al. The pathophysiology of chronic thromboembolic pulmonary hypertension. Eur. Respir. Rev. 26, 160112 (2017).

17. Li, L. et al. Mammalian target of rapamycin overexpression antagonizes chronic hypoxia-triggered pulmonary arterial hypertension via the autophagic pathway. Int. J. Mol. Med. 36, 316-322 (2015).

18. Ogawa, A. et al. Inhibition of mTOR attenuates store-operated Ca2+ entry in cells from endarterectomized tissues of patients with chronic thromboembolic pulmonary hypertension. Am. J. Physiol. Lung. Cell. Mol. Physiol. 297, L666-L676 (2009).

19. Zhao, Y. D. et al. A biochemical approach to understand the pathogenesis of advanced pulmonary arterial hypertension: metabolomic profiles of arginine, sphingosine-1-phosphate, and heme of human lung. PLoS ONE 10, e0134958 (2015).

20. Gilkes, D. M., Bajpai, S., Chaturvedi, P., Wirtz, D. \& Semenza, G. L. Hypoxia-inducible factor 1 (HIF-1) promotes extracellular matrix remodeling under hypoxic conditions by inducing P4HA1, P4HA2, and PLOD2 expression in fibroblasts. J. Biol. Chem. 288, 10819-10829 (2013).

21. Xu, R. et al. NC1 domain of human type VIII collagen (Alpha 1) inhibits bovine aortic endothelial cell proliferation and causes cell apoptosis. Biochem. Biophys. Res. Commun. 289, 264-268 (2001). 
22. Huang, L. et al. Transgelin as a potential target in the reversibility of pulmonary arterial hypertension secondary to congenital heart disease. J. Cell. Mol. Med. 22, 6249-6261 (2018).

23. Machado, R. F. \& Gladwin, M. T. pulmonary hypertension in hemolytic disorders: pulmonary vascular disease: the global perspective. Chest 137(6 Suppl), 30S-38S (2010).

24. Minneci, P. C. et al. Hemolysis-associated endothelial dysfunction mediated by accelerated NO inactivation by decompartmentalized oxyhemoglobin. J. Clin. Invest. 115, 3409-3417 (2005).

25. Mathew, R., Huang, J., Wu, J. M., Fallon, J. T. \& Gewitz, M. H. Hematological disorders and pulmonary hypertension. World J. Cardiol. 8, 703 (2016).

26. Cai, H. \& Harrison, D. G. Endothelial dysfunction in cardiovascular diseases: the role of oxidant stress. Circ. Res. 87, 840-844 (2000).

27. Incalza, M. A. et al. Oxidative stress and reactive oxygen species in endothelial dysfunction associated with cardiovascular and metabolic diseases. Vascul. Pharmacol. 100, 1-19 (2018).

28. Kidd, P. M. Glutathione: systemic protectant against oxidative and free radical damage. Altern Med Rev. 1, 155-176 (1997).

29. McLeay, Y., Stannard, S., Houltham, S. \& Starck, C. Dietary thiols in exercise: oxidative stress defence, exercise performance, and adaptation. J. Int. Soc. Sports Nutr. 14, 12 (2017).

30. Tardiolo, G. et al. Overview on the effects of N-acetylcysteine in neurodegenerative diseases. Molecules 23, E3305 (2018).

31. Higashi, Y., Pandey, A., Goodwin, B. \& Delafontaine, P. Insulin-like growth factor-1 regulates glutathione peroxidase expression and activity in vascular endothelial cells: implications for atheroprotective actions of insulin-like growth factor-1. Biochim. Biophys. Acta 1832, 391-399 (2013).

32. Yang, W. S. et al. Regulation of ferroptotic cancer cell death by GPX4. Cell 156, 317-331 (2014).

33. Zhang, Y., Handy, D. E. \& Loscalzo, J. Adenosine-dependent induction of glutathione peroxidase 1 in human primary endothelial cells and protection against oxidative stress. Circ. Res. 96, 831-837 (2005).

34. Barroso, M. et al. Inhibition of cellular methyltransferases promotes endothelial cell activation by suppressing glutathione peroxidase 1 protein expression. J. Biol. Chem. 289, 15350-15362 (2014).

35. Forgione, M. A. et al. Cellular glutathione peroxidase deficiency and endothelial dysfunction. Am. J. Physiol. Circ. Physiol. 282, H1255-H1261 (2002).

36. Konior, A., Schramm, A., Czesnikiewicz-Guzik, M. \& Guzik, T. J. NADPH Oxidases in vascular pathology. Antioxid. Redox Signal. 20, 2794-2814 (2014).

37. Kirsch, M. \& De Groot, H. NAD(P)H, a directly operating antioxidant?. FASEB J. 15, 1569-1574 (2001).

38. Skvarilová, M., Bulava, A., Stejskal, D., Adamovská, S. \& Bartek, J. Increased level of advanced oxidation products (AOPP) as a marker of oxidative stress in patients with acute coronary syndrome. Biomed. Pap. Med. Fac. Univ. Palacky. Olomouc. Czech. Repub. 149, 83-87 (2005).

39. Vona, R. et al. Oxidative stress in the pathogenesis of systemic scleroderma: an overview. J. Cell. Mol. Med. 22, 3308-3314 (2018).

40. Fakhruddin, S., Alanazi, W. \& Jackson, K. E. Diabetes-induced reactive oxygen species: mechanism of their generation and role in renal injury. J. Diabetes Res. 2017, 1-30 (2017).

41. Kattoor, A. J., Pothineni, N. V. K., Palagiri, D. \& Mehta, J. L. Oxidative stress in atherosclerosis. Curr. Atheroscler. Rep. 19, 42 (2017).

42. Granger, D. N. \& Kvietys, P. R. Reperfusion injury and reactive oxygen species: the evolution of a concept. Redox Biol. 6, 524-551 (2015).

43. Forrester, S. J., Kikuchi, D. S., Hernandes, M. S., Xu, Q. \& Griendling, K. K. Reactive oxygen species in metabolic and inflammatory signaling. Circ. Res. 122, 877-902 (2018).

44. Kumari, S., Badana, A. K. \& Malla, R. Reactive oxygen species: a key constituent in cancer survival. Biomark. Insights 13, $1177271918755391(2018)$

45. Nukala, S. B. et al. Differentially expressed proteins in primary endothelial cells derived from patients with acute myocardial infarction. Hypertension 74, 947-956 (2019).

46. Cox, J. \& Mann, M. MaxQuant enables high peptide identification rates, individualized p.p.b.-range mass accuracies and proteomewide protein quantification. Nat. Biotechnol. 26, 1367-1372 (2008).

47. Thurmond, P., Yang, J. H., Li, Y., Lerner, L. B. \& Azadzoi, K. M. Structural modifications of the prostate in hypoxia, oxidative stress, and chronic ischemia. Korean J. Urol. 56, 187-196 (2015).

48. Naik, P., Sajja, R. K., Prasad, S. \& Cucullo, L. Effect of full flavor and denicotinized cigarettes exposure on the brain microvascular endothelium: a microarray-based gene expression study using a human immortalized BBB endothelial cell line. BMC Neurosci. 16, 38 (2015).

49. Lyu, Z. et al. PPAR $\gamma$ maintains the metabolic heterogeneity and homeostasis of renal tubules. EBioMedicine. 38, 178-190 (2018).

\section{Author contributions}

O.T., V.S., I.B., V.I.P. conducted the experiments to isolate cells from the patients. O.T., J.A.B, M. Caste., and M.Cas. designed the cellular study and experiment protocol. A.D., G.A. and M.Car. designed the quantitative proteomics approach and supervised the experiment protocols. S.B.N, G.B. and A.A.A conducted the proteomics experiments and data analyses. All authors reviewed the manuscript.

\section{Funding}

The authors acknowledge support from the University of Milan through the APC initiative. The authors acknowledge support from Unitech OMICs, University of Milan through MS raw data' acquisition. This present work was funded by Horizon 2020 program of the European Union-Marie Skłodowska- Curie Actions, Innovative Training Networks (ITN), Call: H2020-MSCA-ITN-2015, Number: 675527-MOGLYNET. Tura-Ceide was the recipient of Marie Curie Post-Doctoral Fellowship Award BIOTRACK: IDIBAPS, Spanish Society of Respiratory Medicine (SEPAR 2013), Catalan Society of Pneumology (SOCAP 2015), Fundación Contra la Hipertensión Pulmonar (FCHP). Barberà was funded by Generalitat de Catalunya (2017-SGR-00617). Cascante was funded by Generalitat de Catalunya (2017SGR1033 from AGAUR and "Icrea Academia Award" from Icrea Foundation) and MINECO-European Commission FEDER funds- "Una manera de hacer Europa" (SAF2017-89673-R). Tura-Ceide, Barberà and Cascante were funded by the Institute of Health Carlos III, Spain (OTC: CP17/00114, PI18/00960; JAB: PI15/00582; MC: CIBEREHD-CB17/04/00023 and PT17/0009/0018). Cofunding was provided by the Fondo Europeo de Desarrollo Regional (FEDER); "Una manera de hacer Europa”.

\section{Competing interests}

The authors declare no competing interests. 


\section{Additional information}

Supplementary Information The online version contains supplementary material available at https://doi. org/10.1038/s41598-021-85004-z.

Correspondence and requests for materials should be addressed to S.B.N. or A.D.

Reprints and permissions information is available at www.nature.com/reprints.

Publisher's note Springer Nature remains neutral with regard to jurisdictional claims in published maps and institutional affiliations.

(c) (1) Open Access This article is licensed under a Creative Commons Attribution 4.0 International cc) License, which permits use, sharing, adaptation, distribution and reproduction in any medium or format, as long as you give appropriate credit to the original author(s) and the source, provide a link to the Creative Commons licence, and indicate if changes were made. The images or other third party material in this article are included in the article's Creative Commons licence, unless indicated otherwise in a credit line to the material. If material is not included in the article's Creative Commons licence and your intended use is not permitted by statutory regulation or exceeds the permitted use, you will need to obtain permission directly from the copyright holder. To view a copy of this licence, visit http://creativecommons.org/licenses/by/4.0/.

(C) The Author(s) 2021 